\title{
Evolución de la gestión en la administración pública hospitalaria: Un estudio de caso de un hospital de segundo nivel en México \\ (The evolution of public health care management: A case study of a second level hospital in Mexico)
}

\author{
José Alfredo Sánchez Aldape \\ Arturo Briseño García* \\ Mónica Lorena Sánchez Limón*
}

\begin{abstract}
This paper analyses the management of Hospital General in Cd. Victoria, Tamaulipas, México, a decentralized public institute from the State Government that started operations on 1985. The first services offered to the local population was medical consultation, core medical specialties, emergency services, hospitalization, and intensive care unit. Within the hospital activities, both administrative and medical the management continuously looked for best practices that allowed it to reach its goals and fulfill its mission. Current literature provides a diversity of strategic tools and models that create value to public health processes making a more efficient use of resources. With this context, this research takes an exploratory case study approach using semi-structured interviews having as key informants the hospital managers since its foundation, allowing explanations on the evolution of the implemented management practices. This paper infers the need to incorporate actions that contribute to a comprehensive and structured professional management.
\end{abstract}

Key words: hospital, integral control panel, strategic management

JEL: H75, H83.

\footnotetext{
- Universidad Autónoma de Tamaulipas. Email: jasanchez@docentes.uat.edu.mx

* Universidad Autónoma de Tamaulipas. Email: abriseno@docentes.uat.edu.mx

- Universidad Nacional Autónoma de México. Email: msanchel@docentes.uat.edu.mx
} 
Resumen. En el presente trabajo se analiza la gestión del Hospital General de Cd. Victoria, Tamaulipas, México, organismo público descentralizado del Gobierno del Estado de Tamaulipas que inició sus operaciones en el año de 1985. Los primeros servicios ofrecidos a la población fueron consulta externa y algunas especialidades troncales, así como el servicio de urgencias, hospitalización y terapia intensiva. Dentro de sus actividades tanto médicas como de gestión se sostuvo la permanente búsqueda de las mejores prácticas administrativas que permitieron alcanzar los objetivos planteados y cumplir la misión para la que fue creado. Actualmente existe diversidad de herramientas y modelos de gestión estratégica que aportan valor a sus procesos haciendo que las organizaciones logren hacer más eficientes sus recursos. Por lo anterior se llevó a cabo estudio exploratorio a esta unidad hospitalaria a través de la realización de entrevistas semiestructuradas, teniendo como informantes a los distintos administradores que tuvo esta organización desde su fundación, lo que explicará la evolución en las acciones gerenciales implementadas. Este estudio infiere la necesidad de incorporar acciones que contribuyan a una gestión profesional integral y estructurada.

Palabras clave: cuadro de mando integral, gestión estratégica, hospital

\section{Introducción}

\section{Antecedentes}

En los últimos años, se han observado transformaciones importantes en el contexto de la administración de las organizaciones públicas y privadas, algunas de ellas están relacionadas con la incorporación de medidas no financieras en los sistemas de información y control tradicional; así como la búsqueda de la confiabilidad y veracidad de la información que se da a conocer a las partes interesadas con todas las métricas internas consideradas en su gestión (Culebro, 2008).

En particular en la gestión pública a partir de la década de 1980, las organizaciones han experimentado el fenómeno de la innovación a través de cambios estructurales y nuevas prácticas administrativas que se concentran en los procesos y resultados de la administración pública, concretamente, el Banco Mundial (1997) se dedicó integralmente a analizar en forma específica el papel del Estado en el proceso de consolidación de las reformas económicas. A partir de ello se identificaron directrices y recomendaciones sobre la función de los gobiernos en países de América Latina y el Caribe (Tabla 1). 
Tabla 1. Recomendaciones del Banco Mundial 1997

\section{Recomendaciones del Banco Mundial a jefes de estado (Informe 1997)}

- Concretar reformas relacionadas con una mayor cobertura en salud.

- Inversión en mejoramiento y ampliación de infraestructura médica. (Instalaciones y equipamiento).

- Inversión en formación de capital humano (médicos y enfermeras).

Fuente: Banco Mundial (1997)

En ese sentido, la mejora de los servicios proporcionados por el sector salud y los nuevos esquemas de gestión en organizaciones públicas, son un tema de gran relevancia para la comunidad académica, los cuerpos de gobierno de las unidades hospitalarias y para quienes diseñan las políticas públicas. Algunos países Iberoamericanos, han observado una tendencia a destinar más recursos económicos al diseño de iniciativas orientadas a propiciar la modernización de los sistemas de salud. En consecuencia, a partir de la década de 1990, empiezo un cambio estructural, el Estado inicia con la implementación de estrategias basadas en la innovación, y a desempeñarse con eficiencia en su gestión, en lo que respecta a la eficiencia, Pardo (2004) argumentó que para administrar con eficiencia en la gestión pública es necesario cambiar las estructuras tradicionales por un modelo contemporáneo de una Nueva Gestión Pública.

\section{La eficiencia en la prestación de la salud en México.}

En México, algunos indicadores de productividad entre 1987 y 1997 señalan que el gasto administrativo de la Secretaria de Salud fue $23.4 \%$ de su gasto total; mientras que en el Instituto Mexicano del Seguro Social (IMSS) fue del $4.49 \%$. En este sentido, el IMSS mostró una mejor eficacia en sus operaciones, comparada con la Secretaria de Salud. Otro indicador básico de productividad es el de la consulta externa por médico, en el caso de la Secretaria de Salud fue de $3.77 \%$ y en el IMSS este mismo indicador fue el del $7.7 \%$, de esta forma se infiere que en base a esos indicadores el IMSS tiene una mejor eficiencia operativa que la Secretaria de Salud, entendiendo por "eficiencia operativa" en el medio hospitalario, la optimización de los tiempos y los recursos disponibles, para cubrir los costos operativos más la 
posibilidad de generar un margen de beneficio sin que esto signifique la práctica de actividad lucrativa (Jaimes, 2004).

En este orden de ideas, Fineberg (2007) señala que muchas naciones fallan en el logro de sus metas básicas en los sistemas de salud como son el acceso universal a ella, alta calidad uniforme y costos razonables, así mismo menciona que desde el año 2004 en México la reforma en salud está centrada básicamente en el programa "Seguro Popular" modalidad que incorpora una atención médica a quienes no cuentan con derecho a la medicina social. De esta forma, se pretende atender los ideales de la revolución y de la declaración constitucional que establece que "el cuidado de la salud es un derecho humano", (artículo 4to de la Constitución Política de los Estados Unidos Mexicanos).

\section{Los compromisos del sector salud en México}

En México, las reformas en salud han permitido la asignación de recursos adicionales aunque se observa un rezago histórico en este concepto por la Secretaría de Hacienda y Crédito Público (Visión Funsalud, 2006), también la Organización Mundial de la Salud (OMS, 2000) ha confirmado a través de la primera evaluación del desempeño de los sistemas de salud, en donde México mostró un nivel intermedio en relación con el grupo de países que forman parte, siendo una de las principales razones la carencia de recursos destinados a la salud. Por lo tanto, se recomendó que para lograr obtener los recursos que permitan solucionar las demandas actuales del sector salud en México era necesario:

- Aumentar la asignación de recursos para aplicarse en la implementación de estrategias innovadoras y sustentables de mejorar la asignación del gasto público en salud.

- Implementación de acciones que permitan una mayor cobertura en salud por los recursos invertidos, lo que se permitirá una oferta de servicios oportunos, seguros y efectivos para asegurar la atención de la salud de los mexicanos.

De ahí que la gestión de las unidades de salud pública, deberían de sustentarse y alinearse fundamentalmente con el Programa Nacional de Desarrollo (PND) así como con el Plan Estatal de Desarrollo (PED) bajo un enfoque innovador que busque mejorar el desempeño de las organizaciones 
públicas al brindar servicios de calidad. En este mismo orden de ideas, Navarro (2008) menciona que el objetivo de toda organización es perdurar y para alcanzar ese propósito los componentes que no deben faltar en toda organización o institución son: la eficiencia (aprovechamiento de recursos), la calidad (mejora de procesos y satisfacción del cliente), la calidez o trato humano al cliente (capacidad de ofrecer en tiempo y en forma los servicios).

Investigaciones realizadas sobre la gestión en las unidades hospitalarias públicas en Chile, Argentina, Ecuador, Colombia, Costa Rica y México (Sojo, 2001) y (Secretaria de Salud, 2006) muestran la vulnerabilidad en sus sistemas de gestión. Por lo que en las unidades hospitalarias es fundamental identificar nuevas áreas de oportunidad, que les permita lograr el mejor desempeño de sus procesos internos. Los procesos al interior de las organizaciones cuentan con una dinámica en la interacción con su entorno, al tratarse de acciones entre usuarios internos y externos, proveedores, acreedores y otras unidades de salud e incluso el mismo Estado. Al crear un sistema de gestión que incluya los elementos necesarios, puede considerarse como una propuesta de solución para que las unidades hospitalarias del sector público logren mantener una continuidad de sus objetivos a mediano y largo plazo, sin que se vean interrumpidos por fenómenos de carácter político-social de su comunidad, logrando así mantener un ritmo progresivo en su evolución y su organización.

Por lo tanto, se observa un área de oportunidad para investigar y ayudar a que exista una buena administración de las unidades hospitalarias, teniendo como punto de partida cumplir la principal misión de prestar servicios de salud dignos a la población y gestionar eficientemente los recursos para ofrecer servicios adecuados en el momento que sean requeridos. De ahí que, en esta investigación, busca responder al siguiente cuestionamiento: ¿cuáles fueron las prácticas de gestión profesional implementadas en el Hospital General de Cd. Victoria durante los primeros cuatro periodos administrativos?

Por lo anterior se realizó estudio exploratorio de las distintas acciones que los administradores en turno desarrollaron en sus diferentes periodos como gestores, con el objetivo de conocer la evolución e innovación en la administración de sus recursos, identificando factores clave que contribuyeron o en su caso inhibieron el mejoramiento de mejores prácticas, métodos y acciones de administración estratégica en la unidad de estudio. 


\section{Revisión de la literatura sobre modelos de gestión}

Los modelos de gestión constituyen en la actualidad una herramienta fundamental para las organizaciones públicas y privadas, mediante las cuales se puede ejercer una administración de recursos optima donde en gran medida los resultados de la misma son satisfactorios.

Generalmente, en su gran mayoría las organizaciones emplean instrumentos tecnológicos, la creación de este tipo de apoyos ha permitido elevar el nivel de eficiencia en el uso y administración de los recursos, logrando con estos apoyos concretar su filosofía organizacional asegurando los objetivos planteados al inicio de un periodo.

La gran mayoría de modelos diseñados para dar soporte a la gestión en las organizaciones, proponen el mejoramiento de campos específicos de la gestión, es decir que a merced de las circunstancias se han diseñado modelos que se enfocan en los recursos humanos, gestión de la calidad, gestión de intangibles, gestión de procesos y gestión financiera.

Los modelos de gestión más utilizados en capital humano se muestran en la Tabla 2.

Tabla 2. Modelos de gestión más utilizados en la gestión de capital humano.

\begin{tabular}{ll}
\hline \multicolumn{1}{c}{ Modelo } & \multicolumn{1}{c}{ Características } \\
\hline Harper y Linch (1992) & $\begin{array}{l}\text { Se desarrolla a partir de un plan estratégico que adopta } \\
\text { los requerimientos de la organización en aspectos de } \\
\text { administración del capital humano. }\end{array}$ \\
Werther y Davis (1982) & $\begin{array}{l}\text { Muestra dependencia entre acciones que se relacionan } \\
\text { con el capital humano de la organización. El modelo no } \\
\text { tiene una proyección estratégica de los recursos } \\
\text { humanos. } \\
\text { Adalberto Chiavenato (1983) }\end{array}$ \\
$\begin{array}{l}\text { Considera los principales procesos de la administración y } \\
\text { se centran en los aspectos relacionados con el capital } \\
\text { humano de la organización. }\end{array}$ \\
\hline
\end{tabular}

Fuente: Elaboración propia

En Gestión de la Calidad y mejora continua de los procesos y cadenas de valor existen algunos modelos que permiten administrar estrategias con objetivos diversos, los más usados se muestran en la Tabla 3. 
Tabla 3. Modelos de gestión más utilizados en la gestión de la calidad y mejora continua

\begin{tabular}{ll}
\hline \multicolumn{1}{c}{ Modelo } & \multicolumn{1}{c}{ Características } \\
\hline Norma ISO & Conjunto de normas sobre calidad y gestión de la calidad establecida \\
& por la Organización Internacional de Normalización (ISO). Aplicable \\
& cualquier organización orientada a producción de bienes o servicios. \\
& Esta norma determina la manera en que una organización opera sus \\
& estándares de calidad, tiempos de entrega y niveles de servicio. \\
Modelo de & Es un modelo que facilita a la organización a medir su posición camino \\
Excelencia de la & a la excelencia en la gestión. Se basa en la autoevaluación, \\
EFQM (1) & identificando claramente puntos fuertes en las áreas de oportunidad, así \\
& como las limitaciones más importantes, para poner programas de \\
& acción con los que se fortalezca la gestión. \\
Seis Sigma & Método de mejoramiento de procesos, focalizada en la disminución de \\
& la variabilidad de los mismos, consiguiendo reducir o eliminar \\
& desperfectos o fallas en la entrega de un producto o servicio al cliente. \\
& Este modelo de gestión en una evolución de las teorías de la calidad de \\
& más éxito TQM (Administración de la calidad total)
\end{tabular}

(1) Modelo Europeo de Excelencia Empresarial, http://www.efqm.org/

Fuente: Elaboración propia

En la administración de los recursos públicos se debe tener una escrupulosa y correcta asignación de los mismos, para lo cual los modelos más conocidos se presentan en la Tabla 4.

Tabla 4. Modelos de gestión más utilizados en la gestión de recursos públicos

\begin{tabular}{ll}
\hline \multicolumn{1}{c}{ Modelo } & \multicolumn{1}{c}{ Características } \\
\hline Nueva & Es un modelo orientado a gestionar instituciones públicas bajo criterios de \\
Gestión & honestidad y transparencia, orientado a la generación de resultados, nuevas \\
Pública & $\begin{array}{l}\text { formas de control, orientación al ciudadano, responsabilidad social y política } \\
\text { del servicio público y profesionalización de la burocracia. Es considerado } \\
\end{array}$ \\
& $\begin{array}{l}\text { como una nueva cultura organizacional en donde se trasladan las buenas } \\
\text { prácticas del sector privado a las instituciones públicas. }\end{array}$ \\
Modelo & Es un modelo orientado a resultados, coincidente en los principios de gestión \\
Ciudadanía & de la calidad en el servicio al ciudadano.
\end{tabular}

Fuente: Elaboración propia 
El modelo de la Nueva Gestión Publica busca satisfacer las necesidades de los ciudadanos a través de una gestión eficiente y eficaz, para lo cual es necesario que las instituciones desarrollen servicios de mayor calidad. Este modelo tiene como características: a) la elaboración estratégica de iniciativas como impulsores de desarrollo, b) la progresiva separación del modelo burocrático hacia una Administración por Resultados, c) la realización de Acciones que agregan valor, d) el Mejoramiento institucional y crecimiento de la confianza en las acciones del Estado y e) la Equidad social.

La literatura presenta otros modelos de gestión afines con el área financiera, entre la diversidad de modelos existen con aplicación específica en las organizaciones, Pedraza y Lavín (2013) muestran en su obra un estudio de los más utilizados en las empresas en los últimos años, en la Tabla 5 se muestran algunos de ellos.

Tabla 5. Modelos de gestión financieros más comunes

\begin{tabular}{|c|c|}
\hline Modelo & Características \\
\hline $\begin{array}{l}\text { Modelo Costo } \\
\text { Volumen Utilidad }\end{array}$ & $\begin{array}{l}\text { Herramienta de apoyo en las decisiones que permite visualizar un } \\
\text { escenario en el cual se encuentran inmersas tres variables que son } \\
\text { las que identifican al modelo; costos, volúmenes y precios. Permite } \\
\text { identificar la magnitud de las dificultades financieras de la } \\
\text { organización y buscar la solución necesaria (Hansen y Mowen, 2003) }\end{array}$ \\
\hline $\begin{array}{l}\text { Modelo } \\
\text { Administración del } \\
\text { Capital de Trabajo }\end{array}$ & $\begin{array}{l}\text { Este modelo propone diversas estrategias que contribuyen a gestionar } \\
\text { eficientemente el capital de trabajo, lo que contribuye a que las partes } \\
\text { interesadas mejoren su percepción en relación con la calidad en la } \\
\text { prestación de sus servicios. (Lavín, 2010) }\end{array}$ \\
\hline $\begin{array}{l}\text { Modelo de Gestión } \\
\text { Económica } \\
\text { Presupuestal }\end{array}$ & $\begin{array}{l}\text { Modelo utilizado en gran parte en las instituciones públicas y } \\
\text { organizaciones con fines no lucrativos, sin embargo, el sector privado } \\
\text { también incorpora en sus sistemas de información los presupuestos } \\
\text { de acuerdo al sector en el cual se desempeña. }\end{array}$ \\
\hline $\begin{array}{l}\text { Modelo de Cuadro } \\
\text { de Mando Integral } \\
\text { (CMI) }\end{array}$ & $\begin{array}{l}\text { Modelo contemporáneo presentado en } 1992 \text { por sus creadores Robert } \\
\text { Kaplan y David Norton, este modelo tiene como objetivo lograr una } \\
\text { gestión estratégica integral, al establecer métricas por medio de } \\
\text { indicadores definidos en un plan estratégico. Las perspectivas que } \\
\text { contempla este modelo son: financiera, clientes, procesos y de } \\
\text { aprendizaje y crecimiento. }\end{array}$ \\
\hline
\end{tabular}

Fuente: Elaboración propia 


\section{Método}

La presente investigación es de tipo mixto, no experimental ya que incluye técnicas de enfoque cuali-cuantitativo, su alcance es exploratorio - descriptivo y se ejecuta a través de la estrategia de investigación de estudio de caso. (Münch y Ángeles, 2007).

En este contexto, Eisenhardt (1991) concibe un estudio de caso como la forma de realizar investigación que permita entender las acciones actuales en escenarios semejantes, combinando distintas técnicas para la recopilación de evidencia con el fin de mostrar, identificar y generar teoría. Mientras que Yin (1994) lo concibe como un análisis en profundidad de un fenómeno contemporáneo dentro de su contexto real; por lo tanto, el método del estudio de caso es especialmente útil cuando los límites entre el fenómeno y el contexto no son muy claros.

\section{Selección del caso de estudio}

Es importante señalar que la selección de casos no se extrae a partir de una muestra representativa de una población como suele suceder bajo el enfoque estadístico, se trata de una muestra teórica en la que el número de casos a elegir depende de que pueda replicarse o extenderse la teoría hasta que se logre su saturación o redundancia (Eisenhardt, 1991).

Como se explicó en el apartado anterior, en esta investigación se realiza un estudio de caso simple, dado las características de la unidad hospitalaria y los objetivos de investigación se pretendió identificar y analizar la gestión que se implementó a lo largo de un proceso de trasformación hacia una dependencia con una nueva forma de gestión, además de determinar los factores clave que permitan mejorar la administración de sus procesos administrativos que más convenga a los objetivos y necesidades de la organización analizada.

De esta manera, el caso de estudio fue elegido a conveniencia (Yin, 1984) tomando en cuenta ciertos requisitos teóricos al momento de su selección, entre los que se destacan: 
- Que la Unidad Hospitalaria corresponda al de Segundo Nivel de atención de los servicios de salud;

- Se localice en la Zona Centro del Estado de Tamaulipas;

- Que haya experimentado etapas de transición hacia la nueva gestión pública. (procesos de centralización y descentralización);

- Haber sido hospital de referencia del Programa del Seguro Popular;

- Contar con al menos más de 10 años de operación después de la transición entre una etapa y la otra, y

- Que tenga diversificación en la población que atiende.

Por lo anterior, se eligió una organización caracterizada por dos aspectos clave en su configuración: una etapa de descentralización (desde su fundación hasta el año 2000) y una etapa de centralización (del año 2000 al 2015). Así como administraciones diferentes en cada una de esas etapas. Las principales características de esta organización se detallan en la Tabla 6. El estudio de caso es un método de investigación que permite la recopilación de información desde diversas fuentes y tipos bien sea cualitativas $y / 0$ cuantitativas; al respecto se menciona por ejemplo los documentos, registros de archivos, entrevistas directas, observación directa, observación de los participantes e instalaciones u objetos físicos.

Al respecto, Yin (1994) permite la utilización de diversas fuentes de datos, cumpliendo con el principio de triangulación para garantizar la validez interna de la investigación. Este principio permite verificar si los datos obtenidos a través de las diferentes fuentes de información guardan relación entre sí (principio de triangulación); es decir, si desde diferentes perspectivas convergen los efectos explorados en el fenómeno objeto de estudio.

Con fundamento en lo anterior, para obtener un análisis en profundidad y enriquecedor, tanto de los procedimientos de gestión estratégicos implementados en cada una de las etapas de administración que experimentó la unidad hospitalaria, como de los factores clave en la recolección de datos. 
Tabla 6. Características de la unidad hospitalaria analizada

\begin{tabular}{|c|c|}
\hline Concepto & Definición \\
\hline Tipo de unidad hospitalaria & De segundo nivel de tipo general \\
\hline Fundación & Año de 1984 \\
\hline Fases organizacionales & $\begin{array}{l}\text { Descentralización } 1984 \text { a } 2000 \\
\text { Centralización } \quad 2000 \text { a } 2015\end{array}$ \\
\hline Visión Actual & $\begin{array}{l}\text { Consiste en proporcionar a la población asistencia, médico - } \\
\text { sanitaria completa, tanto curativa como preventiva y cuyos } \\
\text { servicios llegan hasta el ámbito familiar. }\end{array}$ \\
\hline Valores Organizacionales & $\begin{array}{l}\text { Vocación de servicio, calidez, responsabilidad, trabajo en } \\
\text { equipo, honestidad, eficacia, compromiso y lealtad }\end{array}$ \\
\hline Política de calidad actual & $\begin{array}{l}\text { Institución comprometida a satisfacer las necesidades de salud } \\
\text { en la población sobre la base de trabajo en equipo con espíritu } \\
\text { de servicio y capacitación permanente para lograr la mejora } \\
\text { continua. }\end{array}$ \\
\hline $\begin{array}{l}\text { Servicios } \\
\text { actualmente }\end{array}$ & $\begin{array}{l}\text { sConsulta Externa; medicina familiar y especialidad, Servicio de } \\
\text { Urgencias, Cirugía General y de Especialidad, Hospitalización, } \\
\text { Cuidados Intensivos, Cuidados Coronarios, Neonatología, } \\
\text { Servicios Auxiliares de Diagnostico; Laboratorio, Radiología, } \\
\text { Patología, Hemodinámica, Unidad de Quemados. }\end{array}$ \\
\hline Equipamiento actual & $\begin{array}{l}\text { Tomografía Axial, Hemodinámica, Hemodiálisis, } \\
\text { Plaquetoféresis, Instrumental para Trasplantes de Órganos. } \\
\text { (Bomba de circulación extracorpórea) }\end{array}$ \\
\hline Tamaño actual & $\begin{array}{l}231 \text { camas censables } \\
56 \text { camas no censables }\end{array}$ \\
\hline Jurisdicción sanitaria & No. 1 \\
\hline Recursos Humanos & De referencia estatal \\
\hline
\end{tabular}

Fuente: Elaboración propia

La exploración y análisis se realizó en dos etapas las cuales se detallan a continuación. 
Etapa 1: Análisis de la gestión en la unidad hospitalaria en el marco de transición experimentada hacia un modelo de nueva gestión pública.

Esta etapa tuvo como propósito analizar la administración en los distintos ciclos de vida de la unidad hospitalaria desde cuatro enfoques (Servicio al Usuario, Procesos Internos, Finanzas y Gestión del Conocimiento), lo que permitió identificar las decisiones implementadas por los administradores responsables en periodo de su administración.

El trabajo de campo en esta etapa tuvo como objetivo fundamental explorar como se llevó a cabo la gestión estratégica de los procesos por los directivos en las diferentes etapas de gestión (Servicio al Cliente, Procesos Internos, Finanzas y Gestión del Conocimiento) según el ciclo de vida de la organización. Cabe señalar que quienes proporcionaron la información en las entrevistas fueron los cuatro administradores que estuvieron al frente de la unidad hospitalaria, el único requisito para la selección de estos informantes el que hayan tenido oficialmente el cargo de director administrativo y que hayan aceptado participar en la entrevista.

El instrumento que se aplicó para esta etapa del trabajo de campo fue la entrevista semi estructurada, que toma como referencia para su construcción, la guía del modelo de Robert Kaplan (1992) que considera cuatro dimensiones (Servicio al cliente, Procesos Internos, Finanzas y Gestión del Conocimiento) en su teoría de BSC. La entrevista se estructuró de la siguiente forma:

Sección 1.- Destinada a datos generales del entrevistado y de la unidad de análisis.

Sección 2.- Agrupa cuatro apartados de acuerdo a las dimensiones o perspectivas del Cuadro de Mando Integral propuesto por Robert Kaplan (1992) que son: la dimensión del servicio al cliente, de los procesos internos, las finanzas y la dimensión de gestión del conocimiento.

Complementariamente, se apoyó a través de otras fuentes de información secundarias, tales como: bases de datos de la organización, informes oficiales u otros reportes institucionales emitidos por la Secretaría de Salud Estatal. En este sentido, basado en la literatura analizada sobre el tema, las principales variables que se consideraron para el análisis se muestran en la Tabla 7. 
Tabla 7. Etapa 1.- Variables de análisis de la gestión estratégica en la unidad hospitalaria de estudio

\begin{tabular}{|c|c|c|c|}
\hline $\begin{array}{l}\text { Dimensión de } \\
\text { Gestión }\end{array}$ & Variables & Indicadores & Fuentes de Información \\
\hline Servicio al cliente & Usuarios & Numero de personal operativos & $\begin{array}{l}\text { Informes y reportes } \\
\text { emitidos por la organización } \\
\text { o por la secretaria de salud }\end{array}$ \\
\hline Servicio al cliente & $\begin{array}{l}\text { El grado de satisfacción } \\
\text { de los usuarios }\end{array}$ & El número de personal administrativo & $\begin{array}{l}\text { Entrevista } \\
\text { Semiestructuradas a los } \\
\text { Administradores (1) }\end{array}$ \\
\hline Servicio al cliente & Recursos Humanos & $\begin{array}{l}\text { Número de doctores } \\
\text { Sistemas de incentivos y sanciones } \\
\text { Las capacidades (formación de los } \\
\text { empleados) la } \\
\text { Programas de capacitación } \\
\text { Inversión en infraestructura } \\
\text { Número de habitaciones }\end{array}$ & $\begin{array}{l}\text { Entrevista } \\
\text { Semiestructuradas a los } \\
\text { Administradores }{ }^{(1)}\end{array}$ \\
\hline Servicio al cliente & Recursos físicos & $\begin{array}{l}\text { Mantenimiento } \\
\text { Inversión en equipo y tecnología } \\
\text { Tecnologías de información (sistemas) }\end{array}$ & $\begin{array}{l}\text { Entrevista } \\
\text { Semiestructuradas a los } \\
\text { Administradores }{ }^{(1)}\end{array}$ \\
\hline Servicio al cliente & Recursos Tecnológicos & $\begin{array}{l}\text { El número de procesos existentes } \\
\text { Número de procesos clave }\end{array}$ & $\begin{array}{l}\text { Entrevista } \\
\text { Semiestructuradas a los } \\
\text { Administradores (1) }\end{array}$ \\
\hline $\begin{array}{l}\text { Procesos } \\
\text { internos }\end{array}$ & Procesos & $\begin{array}{l}\text { Existencia de planes estratégicos } \\
\text { Tiempos estimados para desarrollarlos. } \\
\text { Capacidades requeridas para } \\
\text { desarrollarlos } \\
\text { Existencia de protocolos } \\
\text { Existencia de evaluación de procesos. }\end{array}$ & $\begin{array}{l}\text { Entrevista } \\
\text { Semiestructuradas a los } \\
\text { Administradores (1) }\end{array}$ \\
\hline $\begin{array}{l}\text { Procesos } \\
\text { internos }\end{array}$ & Evaluación & $\begin{array}{l}\text { Mecanismos para asegurar eficiencia. } \\
\text { Existencia de correcciones } \\
\text { Presupuestos. } \\
\text { Indicadores financieros }\end{array}$ & $\begin{array}{l}\text { Entrevista } \\
\text { Semiestructuradas a los } \\
\text { Administradores (1) }\end{array}$ \\
\hline $\begin{array}{l}\text { Recursos } \\
\text { Financieros }\end{array}$ & Recursos Financieros & $\begin{array}{l}\text { Mecanismos de transferencia de } \\
\text { experiencias } \\
\text { Barreras }\end{array}$ & $\begin{array}{l}\text { Entrevista } \\
\text { Semiestructuradas a los } \\
\text { Administradores }\end{array}$ \\
\hline $\begin{array}{l}\text { Gestión del } \\
\text { conocimiento }\end{array}$ & Gestión del Conocimiento & $\begin{array}{l}\text { Tirado de liderazgo de la dirección } \\
\text { Formación académica o capacidades } \\
\text { Grado de satisfacción de los } \\
\text { empleados. } \\
\text { Clima organizacional adecuado. }\end{array}$ & $\begin{array}{l}\text { Entrevista } \\
\text { Semiestructuradas a los } \\
\text { Administradores }\end{array}$ \\
\hline
\end{tabular}

(1) 4 periodos analizados

Fuente: Elaboración propia 
Etapa 2: Análisis de los factores para mejorar el sistema de gestión estratégica en el marco de la nueva gestión pública.

Etapa que implico el análisis e identificación de los factores considerados clave para mejorar el sistema de gestión a través de una triangulación de fuentes de información, (administrativos: empleados operativos, jefes de departamento y directivos) de los principales procesos de la unidad hospitalaria y del análisis de indicadores de productividad (Yin, 1984) a través de la aplicación de un cuestionario; se recopiló información de aquellos factores importantes considerar en el diseño de un Sistema de Gestión Estratégico para unidades hospitalarias del segundo nivel de atención; el cual permitirá identificar las relaciones existentes entre estos elementos.

Para la recopilación de información en esta etapa, se utilizó la técnica de cuestionario con el levantamiento de información con este instrumento atiende a un estudio transversal, basándose principalmente en la percepción que los empleados, jefes de área y directivos tuvieron en una etapa específica de la vida institucional de la unidad hospitalaria de estudio, particularmente la etapa de centralización (Hernández et al., 2008). Se aplicó el cuestionario a un grupo de veinte informantes, habiendo considerado las siguientes características en su selección; que hayan laborando en todas etapas del a vida institucional de la unidad de estudio, que al momento de responder al cuestionario sean empleados y que sus funciones laborales se relacionen con alguna las siguientes áreas: A.-Servicio al Usuario, B.Procesos Internos, C.-Finanzas, D.-Gestión del Conocimiento (ver Tabla 8).

Se procuró un equilibrio de trabajadores encuestados, habiendo entrevistando a cuatro grupos de cinco trabajadores por cada dimensión. En cuanto a la validez del instrumento, este manifiesta el dominio específico del contenido de lo que se pretende analizar en materia de las variables a medir (Hernández et al., 2008). Para la construcción del cuestionario, se revisó la literatura cuyos constructos atienden a elementos teóricos que hacen énfasis en acciones de gestión estratégica para cuatro enfoques de estudio. (Servicio al usuario, Procesos internos, Finanzas y Gestión del conocimiento). La mayor parte de elementos del cuestionario fueron elaborados a partir del instrumento denominado Sistema de Comprobación Rápida del Concepto TPS (Total Performance Scorecard), basado en Rampersad (2003). 


\section{Tabla 8: Etapa 2.- Variables a identificar en el análisis de la gestión estratégica de la unidad hospitalaria de estudio}

\begin{tabular}{|c|c|c|c|}
\hline Dimensión & Variable & Indicador & Preguntas \\
\hline $\begin{array}{l}\text { Servicios } \\
\text { al usuario(1) }\end{array}$ & R. Humanos & $\begin{array}{l}\text { Servicio / } \\
\text { confiabilidad } \\
\text { y oportuni- } \\
\text { dad }\end{array}$ & $\begin{array}{l}\text { Obtiene el servicio que le prometieron } \\
\text { Mantienen registros sin errores y proporcionan detalle de los } \\
\text { servicios otorgados al usuario. } \\
\text { Existe actitud de servicio y buen trato a los pacientes }\end{array}$ \\
\hline $\begin{array}{l}\text { Servicios } \\
\text { al usuario(1) }\end{array}$ & R. Humanos & Empatía & $\begin{array}{l}\text { Atención individualizada } \\
\text { Trato humano Horario adecuado para consultas }\end{array}$ \\
\hline $\begin{array}{l}\text { Servicios } \\
\text { al usuario(1) }\end{array}$ & R. Humanos & $\begin{array}{l}\text { Trato al } \\
\text { cliente }\end{array}$ & $\begin{array}{l}\text { Profesionalismo y ética en la atención a pacientes. Otorgan trato } \\
\text { amable en la atención. Responden al usuario o a sus familiares con } \\
\text { dominio y conocimiento de la problemática de salud del usuario. }\end{array}$ \\
\hline $\begin{array}{l}\text { Servicios } \\
\text { al usuario(1) }\end{array}$ & Capacidades & Atención & $\begin{array}{l}\text { Grado de conocimiento de cantidad y tipos de usuarios Existencia } \\
\text { de protocolos para atención }\end{array}$ \\
\hline $\begin{array}{l}\text { Proceso } \\
\text { internos }\end{array}$ & Capacidades & Servicio & $\begin{array}{l}\text { Desarrollo de procesos adecuados a las necesidades de los } \\
\text { usuarios Evaluación y revisión continua de los procesos } \\
\text { Incorporación de la medición de la satisfacción como indicador de } \\
\text { mejora en los procesos Existencia de sistemas integrales de } \\
\text { información para establecer medidas preventivas y correctivas }\end{array}$ \\
\hline $\begin{array}{l}\text { Proceso } \\
\text { internos }\end{array}$ & R. Humanos & & $\begin{array}{l}\text { Existencia de personal responsable de procesos Grado de } \\
\text { participación de los empleados operativos en propuestas de mejora } \\
\text { del servicio. Poseen, conocimientos, habilidades y destrezas para } \\
\text { prestar el servicio médico. Existen planes de inducción, } \\
\text { capacitación y actualización. }\end{array}$ \\
\hline $\begin{array}{l}\text { Proceso } \\
\text { internos }\end{array}$ & $\begin{array}{l}\text { R. Instalacio- } \\
\text { nes Físicas }\end{array}$ & Instalaciones & $\begin{array}{l}\text { Cuidado de instalaciones e instrumental Instalaciones agradables. } \\
\text { Mantenimiento adecuado }\end{array}$ \\
\hline $\begin{array}{l}\text { Proceso } \\
\text { internos }\end{array}$ & $\begin{array}{l}\text { R. Tecnoló- } \\
\text { gicos }\end{array}$ & Equipo & Equipamiento profesional Equipo Moderno Mantenimiento adecuado \\
\hline Finanzas & $\begin{array}{l}\text { R. Financie- } \\
\text { ros }\end{array}$ & Coste & $\begin{array}{l}\text { Se considera en la planeación financiera a largo plazo La asignación } \\
\text { de recursos guardan alineación con la normatividad institucional o } \\
\text { gubernamental. } \\
\text { Existencia de un comité de vigilancia de costos implicados en la } \\
\text { unidad hospitalaria Establecimiento de medidas correctivas. Existe } \\
\text { disposición alguna que exija a la institución logra alineación } \\
\text { financiera en el uso de recursos } \\
\text { Establecimiento de mecanismos adecuados para la generación de } \\
\text { ingresos en base a las políticas de la unidad hospitalaria }\end{array}$ \\
\hline $\begin{array}{l}\text { Gestión del } \\
\text { Conocimiento }\end{array}$ & R. Humanos & $\begin{array}{l}\text { Satisfacción } \\
\text { del emplea- } \\
\text { do }\end{array}$ & $\begin{array}{l}\text { Existencia de sistemas de información sobre las áreas y } \\
\text { responsables de la unidad hospitalaria Grado de tolerancia de } \\
\text { errores cometidos por parte de los empleados operativos y } \\
\text { administrativos de la unidad Grado de contacto y conocimiento de } \\
\text { los responsables Los empleados cuentan con espacios para pensar, } \\
\text { actuar Existencia de mecanismos para identificar el grado de } \\
\text { satisfacción de los empleados de la unidad hospitalaria Grado en el } \\
\text { que los empleados cuentan con buena salud física y mental Grado } \\
\text { de existencia de barreras en la transferencia del conocimiento y } \\
\text { experiencias dentro de la unidad hospitalaria }\end{array}$ \\
\hline
\end{tabular}

\footnotetext{
(1) Servicios al usuario derechohabiente de los servicios de salud, población abierta y convenios.
}

Fuente: Elaboración propia,con base en Parasuraman et al. (1988), Rampersad (2003) y http://www.efqm.org/ 


\section{Resultados}

Ante los desafíos crecientes de las organizaciones y de la necesidad de contar con una gestión profesional que facilite el cumplimiento de objetivos y metas organizacionales en el sector público, particularmente en el ámbito hospitalario, se desarrolló el presente estudio con el objetivo de explorar la evolución de la gestión profesional en una unidad hospitalaria.

Una vez analizados los resultados de la aplicación de los cuestionarios, se contrastaron con las respuestas proporcionadas por cada administrador, las que fueron transcritas y colocadas en una matriz de contenidos de esta forma se realizó triangulación y confirmación de las mismas destacando algunos aspectos relacionados con la personalidad de cada uno de los administradores así mismo de su formación profesional e idiosincrasia al momento de la toma de decisiones en su desempeño como administrador en los periodos correspondientes. En la Tabla 9 se destacan algunos aspectos extraídos de las entrevistas realizadas a los administradores de la unidad de estudio.

Tabla 9: Etapa 2.- Coincidencias y contrastes de variables de las entrevistas semiestructurada hechas a los administradores.

\begin{tabular}{|c|c|c|}
\hline Variables & Coincidencias & Contrastes \\
\hline $\begin{array}{l}\text { Gestión de } \\
\text { Usuarios }\end{array}$ & Ninguna & $\begin{array}{l}\text { Durante la gestión CENTRALIZADA se considera ampliar la base } \\
\text { de clientes incorporándose el convenio del programa del seguro } \\
\text { popular teniendo una gran base de usuarios. }\end{array}$ \\
\hline $\begin{array}{l}\text { Misión de la } \\
\text { organización }\end{array}$ & $\begin{array}{l}\text { Proporcionar la satisfacción a los usuarios } \\
\text { de acuerdo con los recursos y las } \\
\text { capacidades vigentes de la unidad de } \\
\text { estudio. }\end{array}$ & $\begin{array}{l}\text { Fue necesario en la fase de CENTRALIZACIÓN readecuar los } \\
\text { protocolos de suministro de insumos médicos a los pacientes de } \\
\text { población abierta asimismo del mejoramiento del control interno } \\
\text { en la determinación de las cuentas de los pacientes internados. }\end{array}$ \\
\hline $\begin{array}{l}\text { Visión de la } \\
\text { organización }\end{array}$ & $\begin{array}{l}\text { En ninguna de las etapas se define } \\
\text { estratégicamente la visión de la unidad } \\
\text { hospitalaria y como consecuencia tampoco } \\
\text { se manifiesta acción estratégica que permita } \\
\text { llevar a cabo a la organizacional al } \\
\text { cumplimiento de su visión. }\end{array}$ & Ninguna \\
\hline $\begin{array}{l}\text { Principales } \\
\text { fortalezas }\end{array}$ & Ninguna & $\begin{array}{l}\text { En la etapa de DESCENTRALIZACIÓN se mostró un grado de } \\
\text { mayor eficiencia, en las iniciativas estratégicas relacionadas con } \\
\text { la gestión del área privada de hospitalización. }\end{array}$ \\
\hline $\begin{array}{l}\text { Principales } \\
\text { debilidades }\end{array}$ & Ninguna & $\begin{array}{l}\text { Se debió básicamente a la formación profesional de los gestores, } \\
\text { toda vez que en ambas etapas existieron problemáticas diversas, } \\
\text { pero quienes tuvieron mejores herramientas administrativas para } \\
\text { mejorar la operatividad de la organización fue sin duda la etapa } \\
\text { de CENTRALIZACIÓN. }\end{array}$ \\
\hline
\end{tabular}


Tabla 9: cont.

\begin{tabular}{|c|c|c|}
\hline Variables & Coincidencias & Contrastes \\
\hline $\begin{array}{l}\text { Importancia a la } \\
\text { calidad en el } \\
\text { servicio }\end{array}$ & Ninguna & $\begin{array}{l}\text { En la etapa de DESCENTRALIZACIÓN sede aborda la calidad } \\
\text { como un simple concepto teórico en la etapa de CENTRALI- } \\
\text { ZACIÓN se implementan una serie de medidas que mejoran la } \\
\text { calidad en el servicio camino a la eficiencia de los procesos con } \\
\text { acciones de monitoreo en la satisfacción de los usuarios }\end{array}$ \\
\hline $\begin{array}{l}\text { Tipo de } \\
\text { Administración }\end{array}$ & Ninguna & $\begin{array}{l}\text { Durante la administración en la etapa DESCENTRALIZADA se } \\
\text { utilizó el modelo de administración por objetivos, mientras que en } \\
\text { la administración de la fase CENTRALIZADA se utilizaron } \\
\text { contemporáneos modelos de control y el uso de algunos } \\
\text { indicadores financieros. }\end{array}$ \\
\hline $\begin{array}{l}\text { Percepción del } \\
\text { administrador de la } \\
\text { productividad en su } \\
\text { gestión }\end{array}$ & Ninguna & $\begin{array}{l}\text { En la etapa de DESCENTRALIZACIÓN los entrevistados } \\
\text { manifiesta su productividad con buena calidad mientras que la } \\
\text { etapa de CENTRALIZACIÓN señala con argumentos que hay } \\
\text { muchas formas de medir la calidad, depende de cuál sea el } \\
\text { propósito en los resultados analizados }\end{array}$ \\
\hline $\begin{array}{l}\text { Gestión de recursos } \\
\text { tecnológicos }\end{array}$ & Ninguna & $\begin{array}{l}\text { Durante la etapa de DESCENTRALIZACIÓN se decide invertir en } \\
\text { equipo de cómputo para eficientar procesos administrativos; en la } \\
\text { fase de CENTRALIZACIÓN se decide invertir en tecnología bio- } \\
\text { médica de vanguardia para mejorar la calidad del servicio médico. }\end{array}$ \\
\hline $\begin{array}{l}\text { Uso de indicadores } \\
\text { durante su gestión }\end{array}$ & $\begin{array}{l}\text { El uso de indicadores derivados del proceso } \\
\text { de análisis de los estados financieros } \\
\text { básicos. }\end{array}$ & $\begin{array}{l}\text { Se complementan el uso de los indicadores de los estados } \\
\text { financieros con los del sistema de gestión de la calidad. }\end{array}$ \\
\hline $\begin{array}{l}\text { Gestión de } \\
\text { infraestructura } \\
\text { física }\end{array}$ & $\begin{array}{l}\text { Durante la fase de descentralización no se } \\
\text { le dio interés al mejoramiento de la } \\
\text { infraestructura médica y administrativa. }\end{array}$ & $\begin{array}{l}\text { En la fase de centralización se dio inicio al mejoramiento de la } \\
\text { infraestructura dando inicio por las áreas administrativas, servicio } \\
\text { médico privado y perímetro del hospital. }\end{array}$ \\
\hline $\begin{array}{l}\text { Gestión de los } \\
\text { recursos financie- } \\
\text { ros }\end{array}$ & $\begin{array}{l}\text { Se manifestarán en un inicio periodos de } \\
\text { bonanza con la deficiente estrategia para la } \\
\text { recuperación de las cuentas y documentos } \\
\text { por cobrar provocando escases de recursos } \\
\text { en etapas distintas. }\end{array}$ & $\begin{array}{l}\text { Se llevará a cabo una administración de los recursos financieros } \\
\text { más elaborada con el uso de técnicas proyecciones de flujos de } \\
\text { efectivo y medidas de control interno en la recuperación de la } \\
\text { cartera de clientes }\end{array}$ \\
\hline $\begin{array}{l}\text { Percepción del } \\
\text { aprovechamiento } \\
\text { de los recursos de } \\
\text { la organización. }\end{array}$ & Ninguna & $\begin{array}{l}\text { Durante la etapa de es de descentralización se argumenta la } \\
\text { ausencia de indicadores que muestren la evolución en el } \\
\text { aprovechamiento de sus recursos, mientras que la fase de } \\
\text { centralización se dio inicio al uso de indicadores y de modelos } \\
\text { administrativos que apoyaban en la gestión de la unidad médica. }\end{array}$ \\
\hline $\begin{array}{l}\text { Formación y } \\
\text { experiencia } \\
\text { profesional }\end{array}$ & $\begin{array}{l}\text { Tres de los administradores encuestados } \\
\text { son de formación profesional de contador } \\
\text { público y la de los administradores de la } \\
\text { etapa de centralización además contaban } \\
\text { con una maestría en finanzas, lo que de } \\
\text { alguna forma contribuyó en una } \\
\text { diferenciación de las acciones realizadas en } \\
\text { sus periodos. }\end{array}$ & Ninguna \\
\hline $\begin{array}{l}\text { Número de } \\
\text { empleados en su } \\
\text { gestión }\end{array}$ & Ninguna & $\begin{array}{l}\text { Se observa un crecimiento muy pronunciado de la plantilla del } \\
\text { personal respecto a la última etapa de gestión. }\end{array}$ \\
\hline $\begin{array}{l}\text { Gestión del } \\
\text { aprendizaje y } \\
\text { crecimiento del } \\
\text { capital humano }\end{array}$ & $\begin{array}{l}\text { Este aspecto representó coincidir en ambas } \\
\text { etapas debido al destinar recursos } \\
\text { financieros para la capacitación y } \\
\text { entrenamiento de los trabajadores }\end{array}$ & Ninguna \\
\hline $\begin{array}{l}\text { Gestión del clima } \\
\text { organizacional }\end{array}$ & $\begin{array}{l}\text { En ambas etapas el personal de la } \\
\text { organización se alineó a la normatividad } \\
\text { establecida teniendo como estrategia } \\
\text { focalizar el servicio en los usuarios. }\end{array}$ & $\begin{array}{l}\text { En la medida en que el número de personal crecía, el clima } \\
\text { laboral se ser volcaba más complicado lo que le ocasionaba } \\
\text { discrepancias en las decisiones relacionadas con la operación de } \\
\text { áreas finales de servicio }\end{array}$ \\
\hline
\end{tabular}

Fuente: Elaboración propia 


\section{Discusión}

De la Tabla 9 se desprenden las siguientes reflexiones:

Durante la etapa de DESCENTRALIZACIÓN se presentó la oportunidad de la firma del convenio de derechohabientes del Seguro Popular sin antes haber realizado un diagnóstico sobre la capacidad instalada de la unidad hospitalaria al momento del acuerdo con la dependencia federal lo cual en el corto plazo originó la saturación de los servicios sin que haya existido un plan estratégico que resolviera a fondo esta situación en el mediano plazo.

Otro de los aspectos destacados en la evolución de la gestión, fue el cambio en el control de los suministros y medicamentos a los hospitalizados, con el propósito de administrar adecuadamente el capital de trabajo de la institución, disminuyendo con esto la descapitalización en la recuperación de las cuotas por cobros de créditos a población abierta por servicios médicos proporcionados.

En la fase DESCENTRALIZACIÓN considerada como una etapa donde existía mayor independencia en la gestión de los recursos de la unidad médica, paradójicamente podemos inferir que la fase de CENTRALIZACIÓN a pesar no tener la libertad en la administración de los recursos mostró mayor profesionalismo de sus gestores y una acertada toma de decisiones, logrando dar cumplimiento a sus acciones con eficiencia y eficacia. De las principales debilidades encontradas, estas se relacionan con el perfil de los administradores quienes no tenían los conocimientos y en su caso el entrenamiento especializado necesario que les permitiera solucionar diversos aspectos en su administración.

En los aspectos de calidad del servicio, en la fase de CENTRALIZACION se ha venido mejorando, sin embargo se percibe que su clima organizacional requiere de una comunidad más comprometida, involucrada y motivada que favorezcan los estándares de calidad exigidos por los usuarios.

Las gestiones relacionadas con la compra de tecnologías de la información observaron algunos contrastes, particularmente en la fase de DESCENTRALIZACIÓN se dio preferencia al mejoramiento tecnológico en las áreas administrativas destinadas a la atención al público, mientras que en la fase de CENTRALIZACIÓN se decide invertir mayormente en la 
adquisición de tecnología biomédica, en ese sentido lo relacionado con la gestión de infraestructura no fue del interés de los Administradores en las primeras tres gestiones, mientras que en el último de los periodos evaluados se gestionaron suficientes recursos para su mejoramiento.

Por lo anterior es necesario que las organizaciones del sector publico realicen los cambios en sus procesos y sus modelos de gestión con la periodicidad que juzguen pertinente buscando favorezcan y logren la eficiencia y eficacia para alcanzar los resultados planificados estratégicamente.

\section{Conclusión}

En relación a la administración de la unidad de estudio, en los últimos años presentó una evolucion de administración tradicional hacia un modelo de gestión integral que favoreció su eficiencia interna, mejorando el servicio (generación y entrega del mismo).

Como consecuencia, la restructuración en las instituciones del Estado favorece diversas líneas de acción las que se convierten en políticas públicas, como lo han sido en la sociedad contemporánea de la información, la meritocracia, la ética, las mejores prácticas de buen gobierno, la función pública y un gobierno electrónico, entre otros que logre dar cumplimento a su razón de ser con sus demandantes en un marco de responsabilidad y transparencia en el uso de los recursos públicos.

En ese sentido toma gran importancia el perfil de los profesionales que asumen cargos públicos y más cuando se trata de instituciones que cada día requieren de profesionales con conocimientos en el ramo, habilidades y destrezas (sentido común) que eventualmente no se adquieren en las instituciones educativas universitarias, si no que depende de cada individuo, además de una ética profesional y valores que fraguan la gestión de un buen servidor público.

\section{Referencias}

Banco Mundial (1997). Informe sobre el desarrollo mundial. Washington, DC: Banco Internacional de Reconstrucción y Fomento/Banco Mundial.

Chiavenato, I., Villamizar, G. A. \& Aparicio, J. B. (1983). Administración de recursos humanos (Vol. 2). McGraw-Hill. 
Culebro, J. (2008). Atomización del Estado y nuevas formas de control. La introducción de los Convenios de Desempeño en organizaciones pública, Gestión y Política Pública, 17(1), 35-70.

Eisenhardt, K. M. (1989). Building theories from case study research, Academy of Management Review, 14(4), 532 - 550.

Fineberg H. V (2007), Reforma de salud en México: un trabajo que avanza, Revista Salud Pública de México, 49(supl. 1), 10-11.

Hansen \& Mowen (2003). Administración de costos: contabilidad y control. Cd. México: Editorial Thomson,

Harper, S. \& Lynch, J. (1992). Manuales de recursos humanos. Madrid: Ed. Gaceta de los Negocios.

Hernández, R., Fernández, C. \& Baptista, P. (2008). Metodología de la investigación. Cd. México: McGraw-Hill Interamericana.

Jaimes, C. M. (2004). La descentralización del gasto en salud en México: una revisión de sus criterios de asignación. En C. M. Jaimes, Impuestos y gasto público en México desde una perspectiva multidisciplinaria. Cd. México: Miguel Ángel Porrúa.

Kaplan, R. \& Norton, D. (1992). The balanced scorecard - Measures that drive Performance, Harvard Business Review, 70(1), 71.

Lavín, J. (2010). Administración del capital de trabajo: Un enfoque estratégico de calidad en la gestión pública hospitalaria. Cd. México: Pearson.

Münch, L. \& Ángeles, E. (2007). Métodos y técnicas de investigación. Cd. México: Ed. Trillas.

Navarro, R. P. (2008). Hacia una Nueva Dirección de Hospitales. Cd. México: Ed. Trillas.

OMS (2000). Informe sobre la salud en el mundo 2000. Mejorar el desempeño de los sistemas de salud. Ginebra: Organización Mundial de la Salud.

Parasuraman, A.; Zeithaml, V. \& Berry, L. (1988). Servqual: A multi-item scale for measuring consumer perceptions of service quality, Journal of Retailing, 64(1), 12-40.

Pardo, M. C. (2004). De la administración pública a la gobernanza. Cd. México: Editorial El Colegio de México.

Pedraza \& Lavín (2013), Estructura y diseño organizacional: enfoque para la competitividad y alineación estratégica. Cd. México: Pearson.

Rampersad, H. K. (2003). Total performance scorecard; redefining management to achieve performance with integrity. Londres: Routledge.

Secretaria de Salud (2006). Innovación en la gestión hospitalaria en México. El caso de los H.R.A.E. Cd. México: Editorial Sestante.

Sojo, A. (2001). Reformas de gestión en salud en América Latina, Revista de la CEPAL, 74, 139-157.

Visión Funsalud (2012). La Salud en México: 2006-2012. Cd. México: Grafia Editores.

Werther Jr, W. B. \& Davis, K. (1982). Dirección de personal y recursos humanos. Cd. México: Editorial McGraw Hill.

Yin, R.K. (1994). Case study research. Design and methods. Applied social research methods (Vol. 5, 2nd ed.). Newbury Park, CA: Sage. 